\title{
Undulations on the surface of elongated bubbles in confined gas-liquid flows
}

\author{
M. Magnini, ${ }^{*}$ A. Ferrari, and J. R. Thome \\ Laboratory of Heat and Mass Transfer, \\ Ecole Polytechnique Fédérale de Lausanne, EPFL-STI-IGM-LTCM, \\ Station 9, CH-1015 Lausanne, Switzerland \\ H. A. Stone \\ Department of Mechanical and Aerospace Engineering, \\ Princeton University, Princeton, New Jersey 08544, USA
}

(Dated: October 20, 2017)

\begin{abstract}
A systematic analysis is presented of the undulations appearing on the surface of long bubbles in confined gas-liquid flows. CFD simulations of the flow are performed with a self-improved version of the open-source solver ESI OpenFOAM (release 2.3.1), for $\mathrm{Ca}=0.002-0.1$ and $\mathrm{Re}=0.1-1000$, where $\mathrm{Ca}=\mu U / \sigma$ and $\operatorname{Re}=2 \rho U R / \mu$, with $\mu$ and $\rho$ being, respectively, the viscosity and density of the liquid, $\sigma$ the surface tension, $U$ the bubble velocity and $R$ the tube radius. A model, based on an extension of the classical axisymmetric Bretherton theory, accounting for inertia and for the curvature of the tube's wall, is adopted to better understand the CFD results. The thickness of the liquid film, and the wavelength and decay rate of the undulations extracted from the CFD simulations, agree well with those obtained with the theoretical model. Inertial effects appear when the Weber number of the flow $\mathrm{We}=\mathrm{CaRe}=\mathcal{O}\left(10^{-1}\right)$ and are manifest by a larger number of undulation crests that become evident on the surface of the rear meniscus of the bubble. This study demonstrates that the necessary bubble length for a flat liquid film region to exist between the rear and front menisci rapidly increases above $10 R$ when $\mathrm{Ca}>0.01$ and the value of the Reynolds number approaches 1000 .
\end{abstract}

\footnotetext{
*mirco.magnini@epfl.ch
} 


\section{INTRODUCTION}

The steady propagation of a long gas bubble through a viscous liquid within a circular tube is a classical problem in fluid mechanics, since the pioneering studies of Bretherton [1] and Taylor [2], and its interest has been motivated by applications such as flow in porous media, coating processes, and enhanced oil recovery. Under the dominant effect of capillary forces, the elongated bubble traps a thin film of liquid against the channel wall, and the front and rear menisci are separated by a uniform film thickness region; see the schematic in Fig. 1(a). Bretherton [1] utilized a lubrication theory to analyze the flow in the liquid film and concluded that, in the limit that $\mathrm{Ca}=\mu U / \sigma \ll 1$ and $\operatorname{Re}=2 \rho U R / \mu \ll 1$ (with $\mu$ and $\rho$ being, respectively, the viscosity and density of the liquid, $\sigma$ the surface tension, $U$ the bubble velocity and $R$ the tube radius), the thickness of the liquid film $h_{0}$ scales as $h_{0} / R \approx \mathrm{Ca}^{2 / 3}$. Taylor [2] performed experimental liquid film thickness measurements for very viscous fluids and showed that $h_{0} / R$ reached an asymptotic value of about $1 / 3$ when $\mathrm{Ca} \rightarrow 2$. Ever since, the dynamics of the elongated bubble, in particular of its front meniscus, have been investigated extensively, e.g. effects of inertia [3-7], profiles of the bubble [6, 8, 9],

streamlines ahead of the bubble tip $[4,10]$ and pressure drop $[4,8,11,12]$. A summary of the studies that are relevant to the present work $(\operatorname{Re} \gg 1)$ is provided in Table I.

While the literature concerned with the fluid dynamics of the front meniscus of a bubble in confined geometries is relatively vast, the rear meniscus has received far less attention; see Table I. The liquid film thickness decreases monotonically from the bubble nose to the uniform film region, whilst the gas-liquid interface presents stationary undulations near the rear meniscus of the bubble (see Fig. 1(a)). In the limit that $\mathrm{Ca} \ll 1$ and $\operatorname{Re} \ll$ 1, the theoretical analysis of Bretherton [1] indicated that oscillations induce a minimum film thickness of $0.716 h_{0}$, while their wavelength scales as $h_{0} \mathrm{Ca}^{-1 / 3}$ [13]. However, the results of numerical simulations in the $R e \ll 1$ regime showed significant deviations from the theoretical predictions of minimum film thickness and wavelength already at $\mathrm{Ca} \sim$ $10^{-3}-10^{-2}[3,14]$. Furthermore, both experimental [9] and numerical simulations [3, 9, 14] emphasized that, when Re $\gg 1$, the amplitude of the undulations increases, such that they become more apparent, and their wavelength decreases (for fixed $\mathrm{Ca}$ ).

In summary, it has been observed that the characteristic features of the undulations on the surface of elongated, confined bubbles depend on both Ca and Re, but the literature still lacks 
Table I. Selected studies on the dynamics of the front and rear menisci of elongated bubbles in confined gas-liquid flows, which are relevant to the present work. The Weber number is calculated as $\mathrm{We}=\mathrm{CaRe}$.

Front meniscus

\begin{tabular}{|c|c|c|c|}
\hline Reference & Flow conditions & Study performed & Main observations \\
\hline Giavedoni and Saita [4] & $\begin{array}{c}\mathrm{Ca} \leq 1 \\
0<\operatorname{Re} \leq 70\end{array}$ & $\begin{array}{l}\text { Numerical simulations } \\
\text { of the front meniscus }\end{array}$ & $\begin{array}{l}\text { Inertia has only a moderate effect on } \\
\text { the meniscus shape and film thickness }\end{array}$ \\
\hline Aussillous and Quéré [5] & $\begin{aligned} \mathrm{Ca} & \lesssim 1 \\
\mathrm{Re} & \lesssim 10^{3}\end{aligned}$ & $\begin{array}{c}\text { Experimental measurement } \\
\text { of liquid film thickness }\end{array}$ & $\frac{h_{0}}{R} \approx \frac{\mathrm{Ca}^{2 / 3}}{1+\mathrm{Ca}^{2 / 3}-\mathrm{We}}$ \\
\hline Heil [10] & $\begin{array}{c}\mathrm{Ca} \leq 5 \\
0<\operatorname{Re} \leq 280\end{array}$ & $\begin{array}{l}\text { Numerical simulations } \\
\text { of the front meniscus }\end{array}$ & $\begin{array}{l}\text { A sequence of closed vortices develops } \\
\text { ahead of the bubble tip as Re increases }\end{array}$ \\
\hline de Ryck [6] & $\begin{array}{c}\mathrm{Ca} \leq 2 \\
\mathrm{Re} \lesssim 10^{3}\end{array}$ & $\begin{array}{l}\text { Lubrication theory including inertia } \\
\text { applied to the flow in the liquid film }\end{array}$ & $\begin{array}{l}\text { Data of [2] and [5] } \\
\text { well predicted by the model }\end{array}$ \\
\hline Kreutzer et al. [8] & $\begin{aligned} \mathrm{Ca} & \leq 0.04 \\
\operatorname{Re} & \lesssim 10^{3}\end{aligned}$ & $\begin{array}{l}\text { Numerical simulations } \\
\text { of the entire bubble }\end{array}$ & $\begin{array}{l}\text { The pressure drop across the bubble } \\
\text { scales as } \mathrm{Ca}^{2 / 3} \mathrm{Re}^{1 / 3} \sigma / R\end{array}$ \\
\hline Han and Shikazono [7] & $\begin{aligned} \mathrm{Ca} & \leq 0.2 \\
\mathrm{Re} & \lesssim 10^{3}\end{aligned}$ & $\begin{array}{l}\text { Experimental measurement } \\
\text { of liquid film thickness }\end{array}$ & $\frac{h_{0}}{R} \approx \frac{\mathrm{Ca}^{2 / 3}}{1+\mathrm{Ca}^{2 / 3}+f(\mathrm{Ca}, \mathrm{Re})-g(\mathrm{We})}$ \\
\hline
\end{tabular}

Rear meniscus

\begin{tabular}{|c|c|c|c|}
\hline Reference & Flow conditions & Study performed & Main observations \\
\hline Bretherton [1] & $\begin{aligned} \mathrm{Ca} & \leq 0.005 \\
\mathrm{Re} & \ll 1\end{aligned}$ & $\begin{array}{l}\text { Lubrication theory applied to } \\
\text { the flow in the liquid film }\end{array}$ & $\begin{array}{l}\text { Liquid film profiles show oscillations } \\
\text { with minimum thickness of } 0.716 h_{0}\end{array}$ \\
\hline Ratulowski and Chang [13] & $\begin{array}{l}\mathrm{Ca} \leq 0.2 \\
\mathrm{Re} \ll 1\end{array}$ & $\begin{array}{l}\text { Lubrication analysis based on an } \\
\text { arclength-angle formulation }\end{array}$ & $\begin{array}{l}\text { The wavelength of the oscillations } \\
\qquad \text { is } 4 \pi h_{0} /\left[\sqrt{3}(3 \mathrm{Ca})^{1 / 3}\right]\end{array}$ \\
\hline Edvinsson and Irandoust [3] & $\begin{aligned} \mathrm{Ca} & \leq 0.06 \\
\mathrm{Re} & \lesssim 10^{3}\end{aligned}$ & $\begin{array}{c}\text { Numerical simulations } \\
\text { of the entire bubble }\end{array}$ & $\begin{array}{c}\text { The measured wavelength is lower than } \\
\text { predicted by [13]; undulations become more } \\
\text { apparent as Re increases }\end{array}$ \\
\hline Giavedoni and Saita [14] & $\begin{array}{c}\mathrm{Ca} \lesssim 1 \\
0<\mathrm{Re} \leq 70\end{array}$ & $\begin{array}{l}\text { Numerical simulations } \\
\text { of the rear meniscus }\end{array}$ & $\begin{array}{c}\text { Same as }[3] \text {; at fixed } \mathrm{Ca} \text {, } \\
\text { wavelength decreases as Re increases }\end{array}$ \\
\hline
\end{tabular}

a systematic analysis under a wide range of capillary and Reynolds numbers. Furthermore, no theoretical model is yet available to satisfactorily predict their geometrical parameters when Re $\gg 1$. This topic is now of interest to a series of novel engineering applications and scientific problems where the topology of the ripples and their minimum film thickness are more important than the thickness of the uniform film region. For instance, in the removal of colloids from a microchannel by a translating gas-liquid interface, the local thickness of the liquid layer determines the surface cleaning efficiency [15]. In microchannel flow boiling, peaks of the heat transfer performance are observed upon the crests of these undulations 
[16] but, on the other hand, large amplitude oscillations may trigger local liquid film dryout $[17,18]$, which has a negative impact on the performance and life of a micro-evaporator.

The objective of the present work is to perform a systematic analysis of the undulations appearing on the surface of long bubbles in confined gas-liquid flows, in the range $\mathrm{Ca}=$ $0.002-0.1$ and $\operatorname{Re}=0.1-1000$. CFD simulations based on a Volume Of Fluid (VOF) method [19], and a theoretical model based on the work of de Ryck [6], are utilized to generate numerical and theoretical bubble profiles. The parameters characterizing the undulations in both the CFD simulations and the analytical model results are compared, and their trends versus the governing nondimensional groups are investigated. In particular, we show that in the visco-inertial regime the Weber number of the flow, We $=\mathrm{Ca} \operatorname{Re}=2 \rho U^{2} R / \sigma$, which represents the ratio of inertial stresses to capillary stresses, organizes the basic features of the bubble shape and thin film profile. This article is organized as follows: in Section II, a description of the problem is presented; the CFD model is introduced in Section III; the theoretical model is described in Section IV; finally, the results of the analysis are discussed in Section V.

\section{DESCRIPTION OF THE PROBLEM}

An elongated gas bubble translates at a velocity $U$ in a channel of circular cross-section (radius $R$ ) filled with liquid. Figure 1(a) shows a sketch of the flow configuration under analysis, in a reference frame attached to the gas bubble. The flow field and the bubble profile are assumed axisymmetric. In order to describe the thin liquid film, and by analogy with the notation of Bretherton [1], the origin of the vertical axis $y$ is on the tube's wall and its direction is radially inward. The horizontal coordinate $x$ is directed downstream, and the reference $x=0$ will be changed, as convenient, during this work. In these coordinates, the axial and radial velocities are defined as $u$ and $v$, respectively, with $v$ being positive when directed towards the axis of the channel. The walls of the tube have velocity $u=-U$. The thickness of the liquid film surrounding the bubble is $h(x)$.

Following the notation and description of Bretherton, we assume that there exists a region of uniform film thickness, region $C D$ in Fig. 1(a), where the liquid is moving with a uniform velocity $-U$ and the film has thickness $h_{0}$; this feature will be confirmed by numerical simulations. The thin film region is axially bounded by two dynamic menisci 


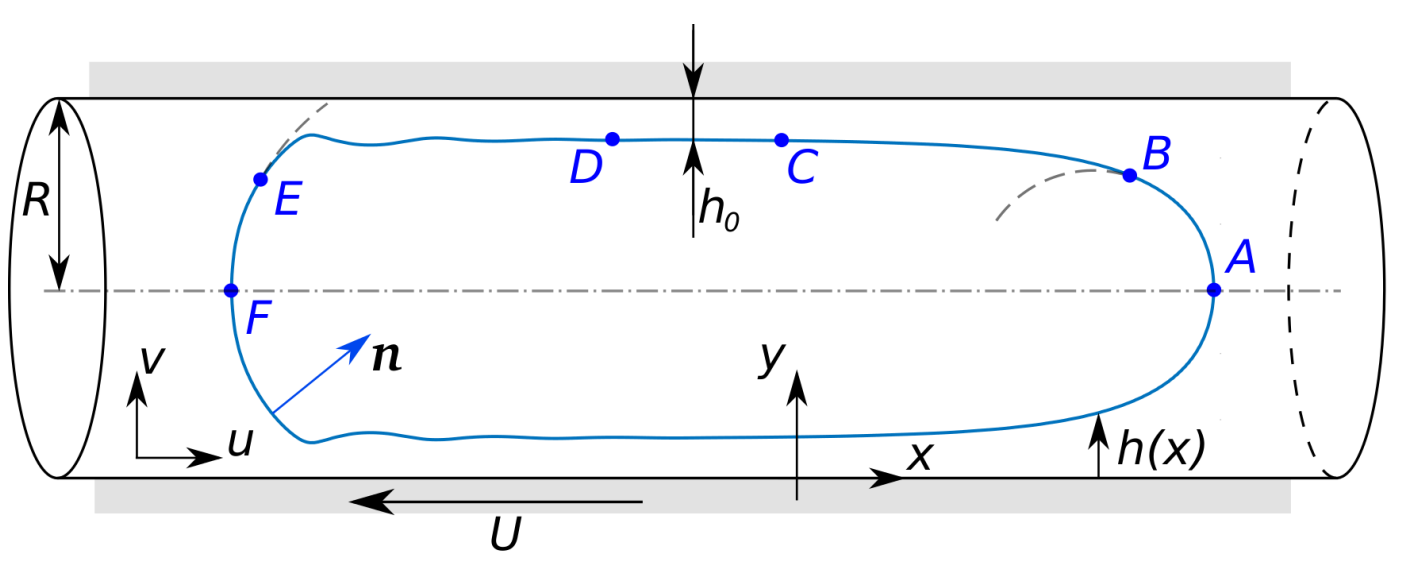

(a)

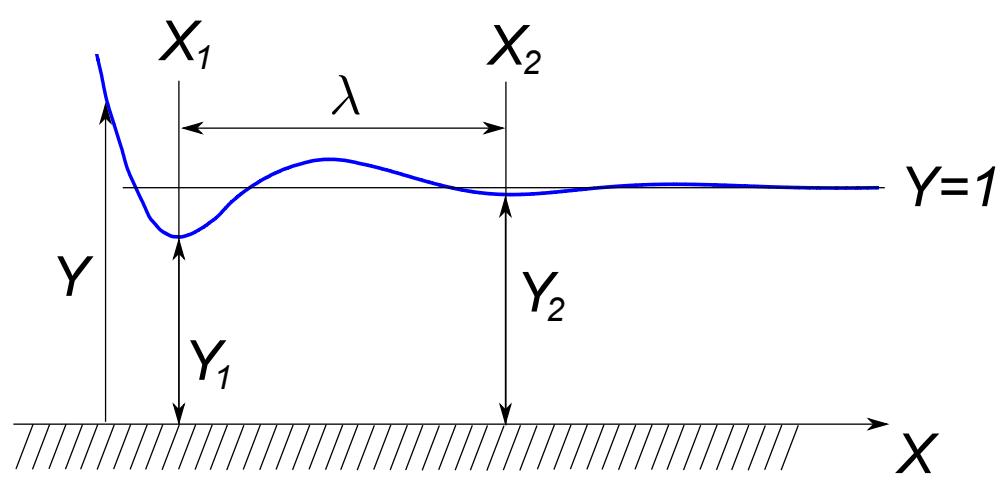

(b)

Figure 1. (a) Sketch of a confined elongated bubble flowing within a tube and notation used in this work. Regions $A B$ and $E F$ represent the front and rear static menisci, respectively. Following the notation of Bretherton, regions $B C$ and $D E$ represent the front and rear dynamic menisci, respectively; region $C D$ represents the uniform film zone. The two circular arcs (grey dashed lines) matching the bubble profile in $B$ and $E$ identify the spheres matching the front and rear static menisci, respectively. (b) Procedure adopted to characterize the decay rate and wavelength of the ripples from the profile of the rear dynamic meniscus.

regions, respectively $B C$ for the bubble front and $D E$ for the bubble back, where the liquid is flowing almost parallel to the wall. In the transition region $B C$, the liquid film thickness increases monotonically from the uniform film region towards the bubble nose. In the rear transition region $D E$, the liquid film thickness oscillates around $h_{0}$. Along the direction of negative $x, h$ reaches a minimum value upon the most upstream crest of the ripple. It eventually grows monotonically as the dynamic meniscus develops towards the terminal 
region $E F$. If $\mathrm{Ca} \ll 1$ and $\mathrm{Re} \ll 1$, the elongated bubble ends with two static menisci regions, respectively $A B$ for the bubble front and $E F$ for the bubble back. Here, all the stresses but surface tension have a negligible effect on the bubble interface, and hence the end caps of the bubble are expected to have spherical shapes, of different radii. The profiles of the matching spheres, extrapolated beyond the respective static menisci, are depicted with grey dashed lines in Fig. 1(a).

We assume that the flow is laminar, steady and incompressible, and that the fluid is Newtonian. The flow in the liquid film is governed by the steady-state Navier-Stokes equations, here written in nondimensional form:

$$
\begin{aligned}
& \widehat{\nabla} \cdot \widehat{\boldsymbol{u}}=0, \\
& \operatorname{Re} \widehat{\boldsymbol{u}} \cdot \widehat{\nabla} \widehat{\boldsymbol{u}}=-\widehat{\nabla} \widehat{p}+\widehat{\nabla}^{2} \widehat{\boldsymbol{u}},
\end{aligned}
$$

where the hats indicate dimensionless entities. The characteristic length scale chosen in Eqs. (1) and (2) is $2 R, \widehat{\boldsymbol{u}}$ represents the fluid velocity scaled on $U, \widehat{\boldsymbol{u}}=\boldsymbol{u} / U$, and $\widehat{p}$ denotes the pressure scaled on the viscous pressure scale, $\widehat{p}=2 R p /(\mu U)$, with $\mu$ being the dynamic viscosity of the liquid. The Reynolds number is defined as Re $=2 \rho U R / \mu$, where $\rho$ is the liquid density. The no-slip condition holds at the wall,

$$
\widehat{u}=-1 \quad \text { and } \quad \widehat{v}=0, \quad \text { at } \quad \widehat{y}=0
$$

At the gas-liquid interface, the boundary conditions are obtained from the dynamic balance of the stresses, here expressed by neglecting the viscous stresses within the gas phase:

$$
-\widehat{p} \boldsymbol{n}+\left(\widehat{\nabla} \widehat{\boldsymbol{u}}+(\widehat{\nabla} \widehat{\boldsymbol{u}})^{T}\right) \cdot \boldsymbol{n}=\frac{1}{\mathrm{Ca}} \widehat{\kappa} \boldsymbol{n}, \quad \text { at } \quad \widehat{y}=\widehat{h}(\widehat{x}),
$$

where $\boldsymbol{n}$ and $\widehat{\kappa}$ indicate the interface normal vector and its dimensionless curvature, respectively. The capillary number is defined as $\mathrm{Ca}=\mu U / \sigma$, where $\sigma$ indicates the surface tension.

Figure 1(b) illustrates the procedure adopted to characterize the decay and wavelength of the ripple appearing at the rear meniscus of the bubble. These details will be described in Section V B. In the figure, the liquid film thickness is rescaled by the uniform liquid film thickness, $Y=h / h_{0}$, and the horizontal coordinate is rescaled by a length $\ell$. The length $\ell$ is on the order of the length of the dynamic menisci, $B C$ and $D E$ in Fig. 1(a), and will be identified below when the lubrication analysis is presented. 


\section{CFD SIMULATIONS}

Numerical simulations of the flow configuration reported in Fig. 1(a) are performed by means of the open-source CFD package ESI OpenFOAM, release 2.3.1. The Volume of Fluid (VOF) [19] based interFoam solver is chosen to capture the interface dynamics. Below, the numerical model is first introduced. Afterwards, the main features of the ripples appearing at the rear meniscus of the bubbles are illustrated with the aid of the simulation results.

\section{A. CFD model}

The solver interFoam solves the unsteady Navier-Stokes equations for a Newtonian fluid and incompressible flow. The two phases are treated as a single mixture fluid with variable properties across the interface, according to the VOF formulation. The surface tension force is included within the momentum equation as a body force according to the Continuum Surface Force (CSF) method of Brackbill et al. [20]. In order to improve the accuracy of the surface tension representation with respect to the original interFoam solver, the interface normal vector and curvature are estimated from a smoothed VOF function field [21] when $\mathrm{Ca}>0.005$ and from a reconstructed level set function [22] when $\mathrm{Ca} \leq 0.005$. Details of the flow equations solved by interFoam and of the numerical methods adopted can be found in Ref. [9].

The flow domain is modelled by adopting a 2D axisymmetric formulation. An elongated bubble is initialized at the upstream end of the domain. At the inlet boundary, liquid enters the flow domain with a fully developed parabolic velocity profile. At the channel wall, a no-slip condition is imposed. At the outlet boundary, the pressure is set to a zero reference value while the velocity gradient along the stream direction is set to zero [23]. The liquid flow rate at the domain inlet, the fluid properties and the initial volume of the bubble for each simulation run are adjusted to match the target values of the capillary and Reynolds numbers, and of the length of the bubble. The liquid to gas density and viscosity ratios are respectively fixed to 1000 and 100 . The CFD simulation is run in time until the bubble translates with a constant velocity.

The computational mesh is made of uniform square cells, which are gradually refined at the wall boundary in order to capture the fluid mechanics within the thin liquid film 
surrounding the bubble. To identify parameters for the simulations a grid independence test was run by utilizing the same mesh arrangements described by Khodaparast et al. [9]. It was observed that the main parameters characterizing the bubble profile (uniform liquid film thickness, wavelength of the undulations and minimum film thickness) for a computational mesh including 40 square cells of uniform size along the radial direction, followed by 10 gradually refined mesh elements in the near wall region, deviated by less than $2 \%$ from those measured with more refined grids. This is the computational grid utilized in the present work (see [9] for more details).

The present numerical model was originally validated by Khodaparast et al. [9]. The authors obtained good agreement for the bubble shape, uniform liquid film thickness and bubble velocity with in-house experimental measurements under a large range of flow capillary numbers $10^{-4}<\mathrm{Ca}<10^{-1}$ and Reynolds numbers $10^{-3}<\operatorname{Re}<10^{3}$, with deviations within the uncertainty band of the experiments ( $2 \%$ for bubble velocity and $10 \%$ for film thickness).

\section{B. Characteristic features of the undulations appearing at the rear meniscus}

We first show the profiles of the bubble obtained with the CFD simulations for $\mathrm{Ca}=0.002$, in Fig. 2 for (a) Re $=1$ and (b) Re $=1000$. The length of the bubble, calculated as $L_{b}=x_{A}-x_{F}$ (refer to Fig. 1(a) for the notation), is varied from $L_{b}=2.6 R$ to $18.6 R$. The profiles of the rear meniscus of the bubble all exhibit undulations of decreasing amplitude towards increasing $x$. The results in Fig. 2 demonstrate that the length of the bubble does not have a significant influence, as long as it is sufficiently longer than the dynamic meniscus length (region $D E$ in Fig. 1(a)), such that a uniform film region is recovered. A bubble length of about $L_{b}=5 R$ seems sufficient to satisfy this criterion for the flow conditions presented in the figure. As a reference, Fig. 2 also includes the profile of the rear meniscus of the bubble obtained by solving the lubrication equation governing the flow in the liquid film according to the low capillary number $(\mathrm{Ca} \ll 1)$ and low Reynolds number $(\mathrm{Re} \ll 1)$ model of Bretherton [1]

$$
Y_{X X X}=3 \frac{(Y-1)}{Y^{3}},
$$




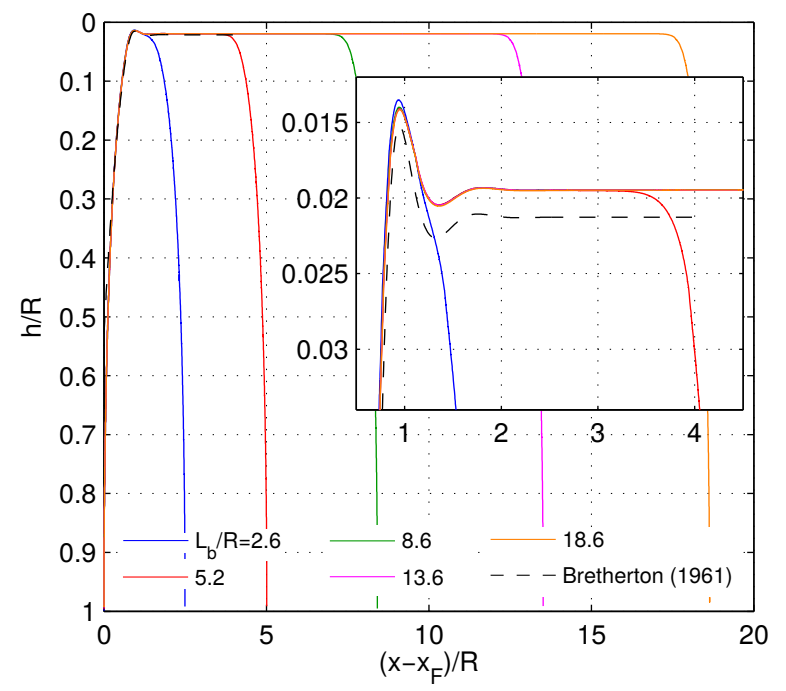

(a)

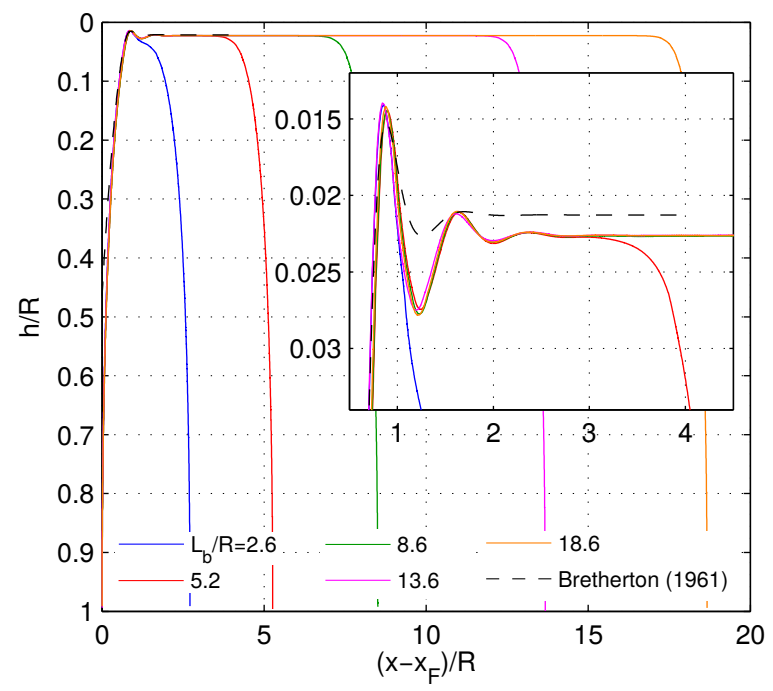

(b)

Figure 2. Profiles of the bubble obtained by CFD simulations for $\mathrm{Ca}=0.002$, (a) $\mathrm{Re}=1$ and (b) Re $=1000$, and different bubble lengths $L_{b} \cdot x_{F}$ locates the intersection between the tube axis and the rear meniscus of the bubble (see Fig. 1(a)).

where $Y=h / h_{0}$ and the subscript indicates a third-order derivative with respect to the nondimensional variable $X$. The length scale $\ell=h_{0} \mathrm{Ca}^{-1 / 3}$ has been used to define $X=$ $x / \ell$. Figure 2(a) shows that for $\mathrm{Re}=1$ the profiles at the bubble back obtained with the CFD simulations agree reasonably well with Bretherton's theory, and the flat film region is recovered after two undulation crests. A deviation of the uniform film thickness of $9.2 \%$ with Bretherton's theory is measured, with $h_{0} / R=0.0195$ in the simulations versus $h_{0} / R=$ $1.34 \mathrm{Ca}^{2 / 3}=0.0213$. This difference can be ascribed to the limited accuracy of Bretherton's law when $\mathrm{Ca}>0.001$. It is observed that, for $\mathrm{Ca}=0.002$, the empirical fit of Aussillous and Quéré [5] to Taylor's data predicts a film thickness of $h_{0} / R=1.34 \mathrm{Ca}^{2 / 3} /\left(1+3.35 \mathrm{Ca}^{2 / 3}\right)=$ 0.0202, with only a $3.6 \%$ deviation from the present CFD data. When Re $=1000$ (Fig. 2(b)), the liquid film thickness increases as reported by many authors $[5,6,8]$, the transition region for the rear meniscus becomes longer, and the undulations become more apparent. Their amplitude increases or, as will be discussed below, their decay rate decreases, and three peaks become clearly visible in Fig. 2(b). The profile corresponding to Eq. (5) does not include the effect of the Reynolds number and therefore it cannot capture the different topology of the undulations. 
In order to emphasize the impact of the Reynolds number on the profile of the bubble back, Fig. 3 presents the results of CFD simulations run at $\mathrm{Ca}=0.002$ and constant bubble length $\left(L_{b} / R \approx 20\right)$ with Re varied from 0.1 to 1000 . It can be seen that for $\operatorname{Re} \leq 100$ there is no appreciable effect of Re and the bubble profiles collapse onto one shape. In these conditions, the shapes of the rear menisci agree well with that calculated according to Bretherton's low Ca and low Re theory. As the Reynolds number is increased above 100, the profiles are modified. The minimum film thickness (measured at the most upstream crest) stays approximately constant, while the amplitude of the undulations increases and more crests appear. Note also that the wavelength, estimated as the horizontal distance between two consecutive peaks, decreases slightly. All of these parameters characterizing the undulations will be studied in detail in Section V by systematically varying the capillary and Reynolds numbers.

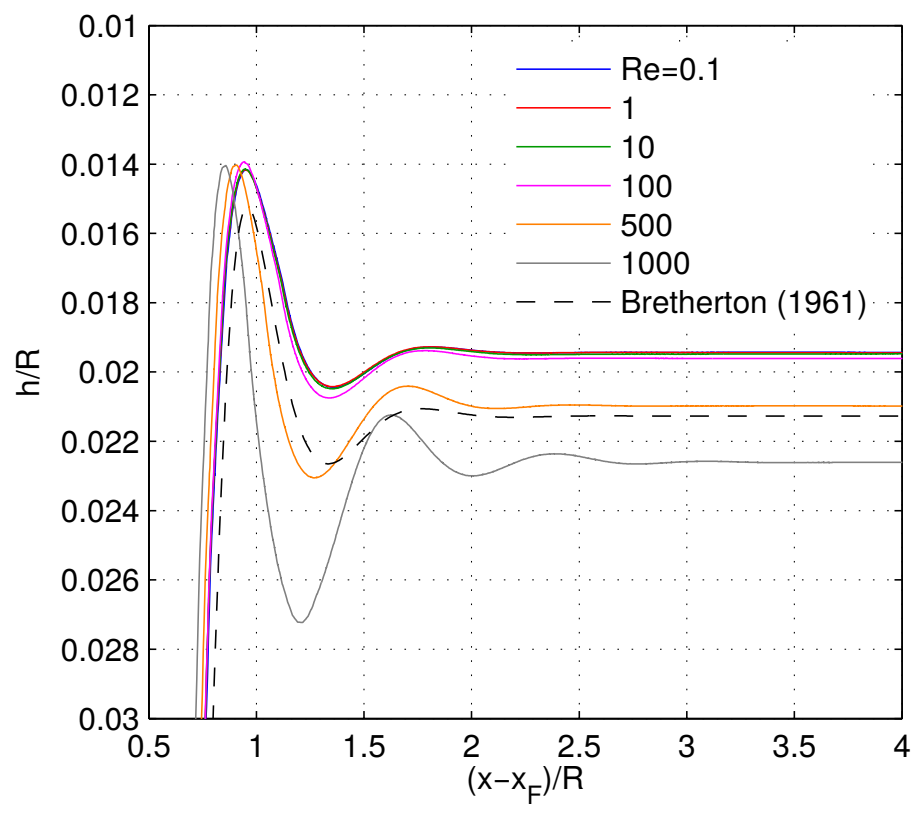

Figure 3. Profiles of the bubble obtained with CFD simulations for $\mathrm{Ca}=0.002$, constant bubble length $L_{b} / R \approx 20$, and different Reynolds numbers. $x_{F}$ locates the intersection between the tube axis and the rear meniscus of the bubble (see Fig. 1(a)). 


\section{THEORETICAL MODEL FOR THE FLOW IN THE LIQUID FILM INCLUD- ING INERTIAL EFFECTS}

The model used in this work to derive theoretical profiles of the rear meniscus of the bubble was originally presented by de Ryck [6], who applied it to investigate the front meniscus of the bubble. Before introducing the complete model, simpler analytical models obtained by increasing levels of complexity starting from Bretherton's theory are briefly reviewed below.

The equations governing the fluid flow in the dynamic menisci regions for the axisymmetric flow configuration depicted in Fig. 1(a) are reported in Appendix A. By assuming that $\delta=h_{0} / \ell \ll 1$, Bretherton [1] simplified the flow equations by retaining only the leading order $\mathcal{O}(1)$ terms, according to the (dimensional) lubrication approximation:

$$
\begin{aligned}
& p_{x}=\mu u_{y y}, \quad p_{y}=0, \\
& \text { with } u_{y}=0, \quad \text { and } p=-\sigma \kappa, \quad \text { at } y=h(x),
\end{aligned}
$$

where the pressure inside the bubble is assumed constant and is set to zero. Under a small slope approximation, $\left|h_{x}\right| \ll 1$,

$$
\kappa=h_{x x}+\frac{1}{R}
$$

By setting $\ell=h_{0} \mathrm{Ca}^{-1 / 3}$, Bretherton obtained a third-order differential equation for the nondimensional profile $Y(X)$, as shown in Eq. (5), which holds as long as $\delta=h_{0} / \ell=$ $\mathrm{Ca}^{1 / 3} \ll 1$ and $\mathrm{Re} \ll 1$.

The effects of inertia in the dynamic menisci regions (BC and DE in Fig. 1(a)) can be introduced in the equations above by retaining the convective term in the $x$-momentum equation, i.e.

$$
\rho\left(u u_{x}+v u_{y}\right)+p_{x}=\mu u_{y y} .
$$

The continuity and momentum equations along $x$ are now replaced by equations integrated 
along the $y$ coordinate from $y=0$ to $y=h(x)$ :

$$
\begin{aligned}
\int_{0}^{h} u_{x} d y+\int_{0}^{h} v_{y} d y & =0 \\
\rho\left(\int_{0}^{h} u u_{x} d y+\int_{0}^{h} v u_{y} d y\right) & =\int_{0}^{h} \sigma \kappa_{x} d y+\int_{0}^{h} \mu u_{y y} d y,
\end{aligned}
$$

where the boundary condition at the free surface Eq. (7) has been used to write $p_{x}=-\sigma \kappa_{x}$. These equations are still difficult to solve because $u$ and $v$ depend on both $x$ and $y$. A method for going further has been proposed by Shkadov [24] and has been afterwards adopted by Esmail and Hummel [25] and Koulago et al. [26] to solve fluid mechanics problems associated with thin liquid film flows in the presence of inertia. The velocity profile is supposed to remain parabolic, as in the purely viscous case, but the effective pressure gradient that induces the flow is taken to be unknown. The velocity profile must satisfy the no-slip condition at the wall and Eq. (7) at the free surface, so that $u(x, y)$ has the form:

$$
u(x, y)=3 F(x)\left[-\frac{y}{h}+\frac{1}{2}\left(\frac{y}{h}\right)^{2}\right]-U,
$$

where the term between square brackets comes from the exact solution in the purely viscous case. Equation (11) can be used in Eq. (10) to replace $u, v$ and their derivatives, thus obtaining a differential equation where both $h(x)$ and $F(x)$ are unknown. The complete derivation is provided in the Supplemental Material. The unknown function $F(x)$ can be written as a function of $h(x)$ by using the flux conservation:

$$
Q=\int_{0}^{h} u(x, y) d y=-\int_{0}^{h_{0}} U d y,
$$

which yields:

$$
F(x)=U\left(\frac{h_{0}}{h}-1\right) .
$$

These steps lead to a nonlinear differential equation for $h(x)$

$$
-p_{x}=\sigma h_{x x x}=3 \mu U \frac{\left(h-h_{0}\right)}{h^{3}}+\frac{1}{5} \rho U^{2}\left(h^{2}-6 h_{0}^{2}\right) \frac{h_{x}}{h^{3}},
$$

which, by adopting the dimensionless coordinates $Y=h / h_{0}$ and $X=x /\left(h_{0} \mathrm{Ca}^{-1 / 3}\right)$, takes the nondimensional form

$$
Y_{X X X}=3 \frac{(Y-1)}{Y^{3}}+\frac{1}{10} \operatorname{Re} \mathrm{Ca}^{1 / 3} H\left(Y^{2}-6\right) \frac{Y_{X}}{Y^{3}},
$$


where $H=h_{0} / R$ is the nondimensional uniform film thickness. The Reynolds number is still defined as $\operatorname{Re}=2 \rho U R / \mu$. The second term on the right-hand side of Eq. (15) is the only additional term compared to Eq. (5), and it introduces the effects of inertia, in the form $\mathrm{Re} \mathrm{Ca}^{1 / 3}$, on the description of the liquid film profile. A closer look at the inertia term actually demonstrates that it is proportional to the Weber number of the flow, We $=\mathrm{Ca}$ Re, as

$$
\mathrm{Re}_{\mathrm{Ca}}{ }^{1 / 3} H \sim \mathrm{Re} \mathrm{Ca}=\mathrm{We}
$$

where Bretherton's law $H \approx \mathrm{Ca}^{2 / 3}$ has been used to express $H$.

This theoretical model is regarded as approximate, because inertia in the $x$-momentum equation is the only first order term added to the leading order Eqs. (6) and (7), while other first order terms, that will be included in the next models developed below, are here neglected in order to keep this first model as simple as possible. Analogous approximations were used by Koulago et al. [26], who derived a lubrication equation formally similar to Eq. (15) to describe the profile of a free interface when a fibre is quickly drawn out of a liquid bath.

Equation (15) can be developed further by retaining all the $\mathcal{O}(\delta)$ terms in the NavierStokes equations, by beginning with

$$
\begin{aligned}
& \rho\left(u u_{x}+v u_{y}\right)+p_{x}=\mu\left(u_{x x}+u_{y y}\right), \quad p_{y}=\mu v_{y y}, \\
& \text { with } u_{y}=0, \quad \text { and } p=-\sigma \kappa+2 \mu v_{y}, \quad \text { at } y=h(x),
\end{aligned}
$$

where the $\mu u_{x x}$ term is included, although being $\mathcal{O}\left(\delta^{2}\right)$, in order to avoid reduction of the order of the boundary condition at the free surface. Integrating Eq. (17) along y, using the boundary conditions in Eq. (18) to express $p_{x}$ and Eq. (11) to write $u$, $v$ and their derivatives, and still writing the interface curvature as indicated in Eq. (8), a nonlinear differential equation is obtained:

$$
Y_{X X X}=3 \frac{(Y-1)}{Y^{3}}+\frac{1}{10} \operatorname{Re} \mathrm{Ca}^{1 / 3} H\left(Y^{2}-6\right) \frac{Y_{X}}{Y^{3}}+3 \frac{\mathrm{Ca}^{2 / 3}}{Y^{3}}\left[\left(\frac{Y}{3}-\frac{3}{2}\right) Y Y_{X X}+3 Y_{X}^{2}\right]
$$

where the last term on the right-hand side arises from the more accurate boundary condition at the free surface. Details of the derivation are given in the Supplemental Material. A similar equation was derived by Esmail and Hummel [25] to describe the film entrained from 
a bath of liquid by the vertical withdrawal of a sheet at high velocity.

Due to the small slope assumption utilized to express the interface curvature, Eq. (19) is expected to work well as long as $\left|h_{x}\right| \ll 1$. Such a hypothesis can be relaxed by considering the complete expression for the interface curvature,

$$
\kappa=\frac{h_{x x}}{\left(1+h_{x}^{2}\right)^{3 / 2}}+\frac{1}{(R-h)\left(1+h_{x}^{2}\right)^{1 / 2}} .
$$

When introducing Eq. (20) into the free surface boundary condition (18), the final differential equation for the film thickness becomes

$$
\begin{aligned}
& Y_{X X X}=3 \mathrm{Ca}^{2 / 3} \frac{Y_{X} Y_{X X}^{2}}{f_{1}}+\frac{H Y_{X}}{\mathrm{Ca}^{2 / 3}(1-H Y)^{2}}\left[\mathrm{Ca}^{2 / 3}(1-H Y) Y_{X X}-H f_{1}\right]+ \\
& +\left\{3 \frac{(Y-1)}{Y^{3}}+\frac{1}{10} \mathrm{Re} \mathrm{Ca}^{1 / 3} H\left(Y^{2}-6\right) \frac{Y_{X}}{Y^{3}}+3 \frac{\mathrm{Ca}^{2 / 3}}{Y^{3}}\left[\left(\frac{Y}{3}-\frac{3}{2}\right) Y Y_{X X}+3 Y_{X}^{2}\right]\right\} f_{1}^{3 / 2},
\end{aligned}
$$

where

$$
f_{1}=1+\mathrm{Ca}^{2 / 3} Y_{X}^{2}
$$

and the term between braces coincides with the right-hand side of Eq. (19). The first and second terms on the right-hand side of Eq. (21) implement the effects of the slope of the interface, and hence Eq. (21) reduces to Eq. (19) when $\left|Y_{X}\right| \ll 1$.

Finally, de Ryck $[6,27]$ introduced the curvature of the tube's wall in the formulation of the problem by retaining the related $\mathcal{O}(\delta)$ term within the $x$-momentum equation,

$$
\rho\left(u u_{x}+v u_{y}\right)+p_{x}=\mu\left(u_{x x}+u_{y y}-\frac{1}{R-y} u_{y}\right) .
$$

The differential equation governing the dynamic meniscus profile then becomes

$$
\begin{aligned}
& Y_{X X X}=3 \mathrm{Ca}^{2 / 3} \frac{Y_{X} Y_{X X}^{2}}{f_{1}}+\frac{H Y_{X}}{\mathrm{Ca}^{2 / 3}(1-H Y)^{2}}\left[\mathrm{Ca}^{2 / 3}(1-H Y) Y_{X X}-H f_{1}\right]+ \\
& +\left\{3 \frac{(Y-1)}{Y^{3}} f_{2 a}+\frac{1}{70} \mathrm{Re} \mathrm{Ca}^{1 / 3} H \frac{Y_{X}}{Y^{3}} f_{2 b}+3 \frac{\mathrm{Ca}^{2 / 3}}{Y^{3}}\left[Y Y_{X X} f_{2 c}+Y_{X}^{2} f_{2 d}\right]\right\} f_{1}^{3 / 2},
\end{aligned}
$$


with

$$
\begin{aligned}
f_{2 a} & =1+H \frac{Y-1}{2} \\
f_{2 b} & =6\left(Y^{2}+Y-9\right)+H \frac{134 Y^{3}-171 Y^{2}-1131 Y+1728}{32}, \\
f_{2 c} & =\frac{7 Y-33}{20}+H \frac{31 Y^{2}-150 Y+99}{120}, \\
f_{2 d} & =\frac{2 Y+31}{10}+H \frac{2 Y^{2}+19 Y-31}{20} .
\end{aligned}
$$

Note that the terms between braces are formally similar to those included in Eq. (21): the first comes from the standard Bretherton problem, the second from the inclusion of inertia, and the third from the shear stress term within the boundary condition at the free surface, Eq. (18). Since all the terms that are $\mathcal{O}(\delta)$ are now retained, the model above should hold as long as $\delta^{2}=\mathrm{Ca}^{2 / 3} \ll 1$. de Ryck [6] validated this model against the experimental film thickness measurements of Taylor [2] for negligible Reynolds numbers and obtained a very good agreement up to $\mathrm{Ca}=2$. The model was also shown to capture the experimental data of Aussillous and Quéré [5] up to $\mathrm{Re}=2000-2500$, when the flow in the experiments likely transitioned to a turbulent flow.

The differential Eq. (24), with Eqs. (22) and (25) are adopted in this work to investigate the profile of the front and rear dynamic menisci of the bubble when varying the capillary and Reynolds numbers. The numerical integration of Eq. (24) is performed by means of the Matlab solver ode45. The integration starts from close to the flat film region using the linearized conditions $Y(X)=1+e^{s X}, Y_{X}(X)=s e^{s X}, Y_{X X}(X)=s^{2} e^{s X}$, where $s$ is the solution (determined numerically by root finding) of the following equation derived by linearization of Eq. (24) [6]:

$$
s^{3}+\mathrm{Ca}^{2 / 3}\left(\frac{39}{10}+\frac{H}{2}\right) s^{2}+\left[\operatorname{ReCa}^{1 / 3} H\left(\frac{3}{5}-\frac{H}{4}\right)+\frac{H^{2}}{\mathrm{Ca}^{2 / 3}(1-H)^{2}}\right] s-3=0 .
$$

This algebraic equation has one real and two complex conjugate roots, and therefore the general solution of the linear problem is

$$
Y(X)=1+\epsilon e^{\beta X}+\gamma_{1} e^{-\alpha X} \cos \left(\frac{2 \pi X}{\lambda}\right)+\gamma_{2} e^{-\alpha X} \sin \left(\frac{2 \pi X}{\lambda}\right)
$$

where $\beta, \alpha$ and $\lambda$ derive from the solution of Eq. (26) and are functions of both Ca and Re. 
The sine term can be eliminated by introducing a new constant $\varphi$,

$$
Y(X)=1+\epsilon e^{\beta X}+\gamma e^{-\alpha X} \cos \left(\frac{2 \pi X}{\lambda}+\varphi\right) .
$$

The procedure for the numerical integration of Eq. (24) at the front and rear menisci is illustrated in the following sections.

\section{A. Numerical integration at the front meniscus}

The integration for the front meniscus starts from close to the uniform film region (point $\mathrm{C}$ in Fig. 1(a)) and proceeds towards $X \rightarrow+\infty$. The linearized initial conditions are:

$$
Y(X=0)=1+\epsilon, \quad Y_{X}(X=0)=\epsilon \beta, \quad Y_{X X}(X=0)=\epsilon \beta^{2}
$$

where in our calculation we take $\epsilon=10^{-4}$.

Compared to the Bretherton problem governed by Eq. (5), the additional terms appearing in the theoretical model used here introduce two difficulties. First, the differential equation for the liquid film profile now depends on the nondimensional uniform film thickness $H$. Second, integration of Eq. (24) shows that $Y_{X}$ and $Y_{X X}$ quickly diverge towards very large values as $Y$ increases. Therefore, the asymptotic matching adopted by Bretherton [1] cannot be used. The procedure to match the film profile in the dynamic region to a static meniscus of spherical shape (point B in Fig. 1(a)) is as follows. For a given set of Ca and Re, there exists a range of values of $H, 0<H \leq H^{*}$, that allow matching the meniscus to a sphere. The critical value $H^{*}$ is the largest $H$ allowing the matching. As an example, Fig. 4(a) plots the different shapes of the front meniscus obtained by numerical integration for $\mathrm{Ca}=0.01$ and $\operatorname{Re}=1000$, and four different values of $H$. Figure $4(\mathrm{~b})$ shows the nondimensional curvature of these interface profiles, $\widehat{\kappa}=\kappa /\left(h_{0} / \ell^{2}\right)$, calculated as

$$
\widehat{\kappa}=\frac{Y_{X X}}{\left(1+\mathrm{Ca}^{2 / 3} Y_{X}^{2}\right)^{3 / 2}}+\frac{H}{\mathrm{Ca}^{2 / 3}(1-H Y)\left(1+\mathrm{Ca}^{2 / 3} Y_{X}^{2}\right)^{1 / 2}},
$$

and that of the matching spheres, $\widehat{\kappa}_{s p h}$, calculated as

$$
\widehat{\kappa}_{s p h}=\frac{2 H}{\mathrm{Ca}^{2 / 3}(1-H Y)\left(1+\mathrm{Ca}^{2 / 3} Y_{X}^{2}\right)^{1 / 2}} .
$$

It can be seen that, for each value of $H<H^{*}=0.0642$, there is always one point where $\widehat{\kappa}=\widehat{\kappa}_{s p h}$. This represents the curvature matching point, point B in Fig. 1(a). As $H$ is 


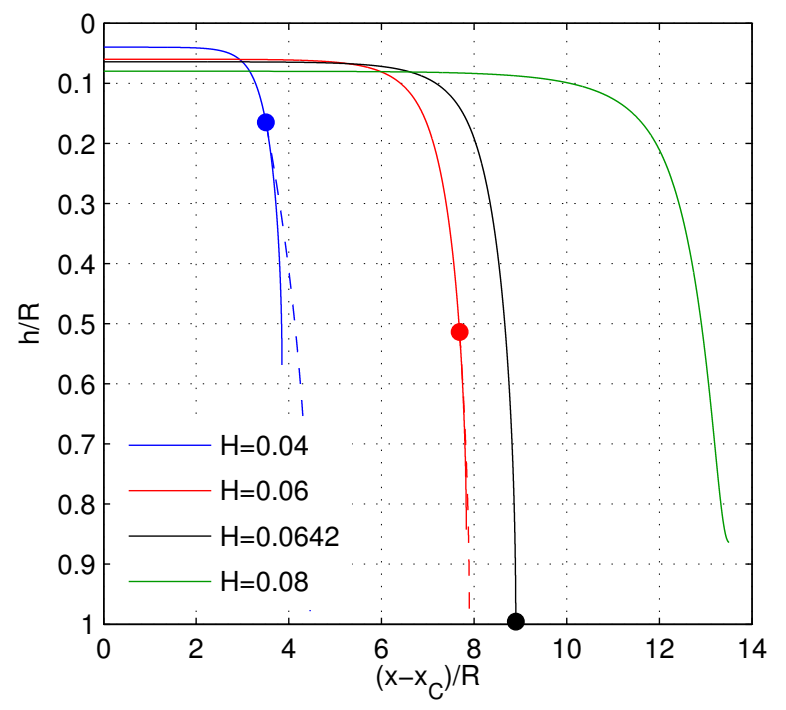

(a)

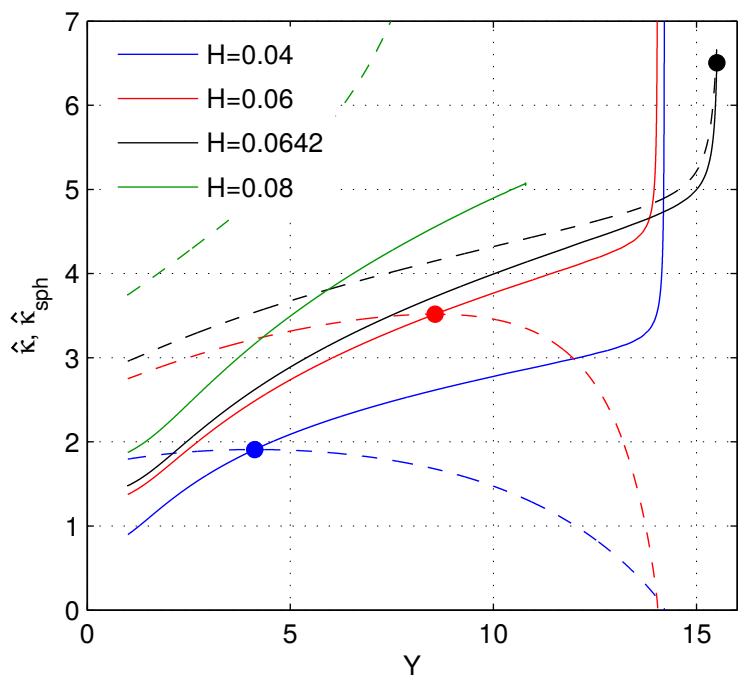

(b)

Figure 4. (a) Profiles of the front meniscus (solid lines) and of the matching sphere (dashed lines), (b) nondimensional curvature of the front meniscus (solid lines) and of the matching sphere (dashed lines), for $\mathrm{Ca}=0.01$ and $\mathrm{Re}=1000$ and different values of $H$. The full symbols identify the curvature matching points for each profile (B in Fig. 1(a)). $x_{C}$ identifies the beginning of the dynamic meniscus at the front of the bubble. No matching is possible for $H=0.08$. The profiles in (a) are not to scale.

increased closer to $H^{*}$, there exists a range of positions where the two curvatures overlap in Fig. 4(b), while the matching point in Fig. 4(a) (full marker) moves towards larger $Y$, and hence closer to the tube axis. Also, note from Fig. 4(a) that the interface profile obtained with $H^{*}=0.0642$ is the only one for which the numerical integration proceeds until the channel axis, where $Y=1 / H$. Instead, when $H<H^{*}$ it is observed that $Y_{X}$ and $Y_{X X}$ diverge to very large values when $Y<1 / H$, thus interrupting the calculation before the channel axis is reached.

de Ryck [6] compared this critical value $H^{*}$ with the experimental measurements of Taylor [2] and Aussillous and Quéré [5] for a wide range of Ca and Re and obtained very good agreement. In this work, we compared the critical value of $H$ extracted from the theoretical model and the film thickness measured in our CFD simulations in the range $\mathrm{Ca}=0.002-0.1$ and $\mathrm{Re}=0.1-1000$. The comparison is presented in Fig. 5. The 


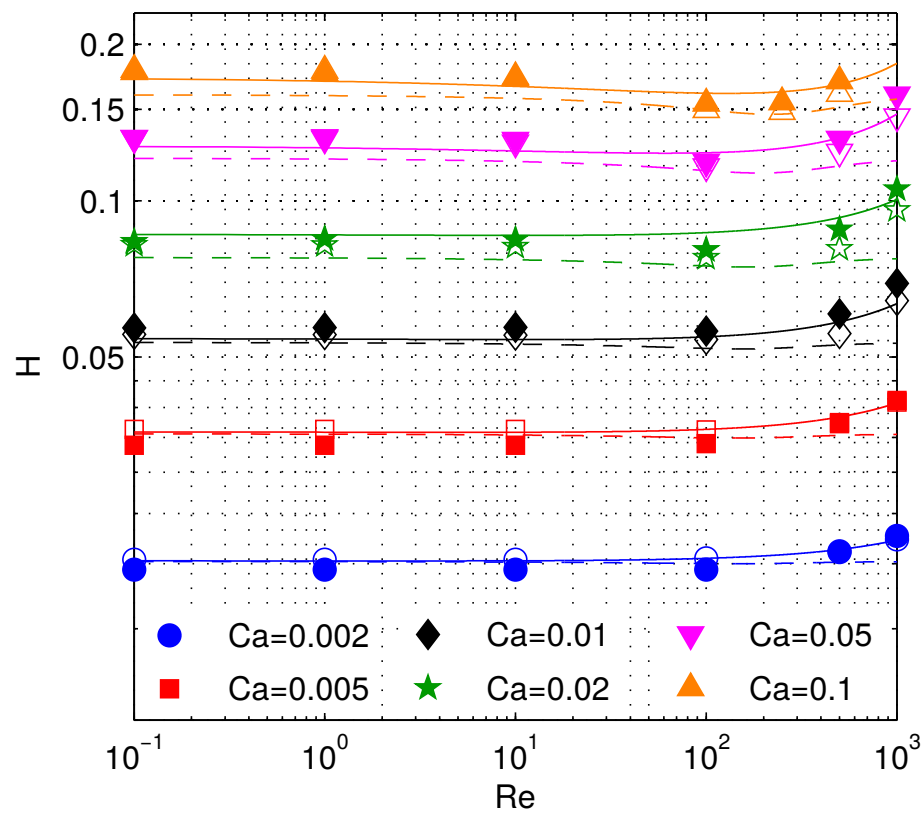

Figure 5. Nondimensional liquid film thickness obtained by the CFD simulations (full symbols), by the theoretical model based on Eq. (24) (open symbols), and predictions given by the empirical correlations of Han and Shikazono [7] (solid lines) and Langewisch and Buongiorno [11] (dashed lines).

bubble length in all of the simulations is about $20 R$. It can be seen that the values of the maximum nondimensional film thickness $H$ that allow matching the bubble nose to a sphere extracted from the theoretical model are in very good agreement with the CFD results, with an average deviation of $3.9 \%$. Figure 5 also shows the predictions given by the empirical correlations of Han and Shikazono [7] and Langewisch and Buongiorno [11], which exhibit deviations from the present CFD data of, respectively, $4.2 \%$ and $8.5 \%$. Although these correlations were obtained by fitting large experimental [7] and numerical [11] databases, they do not perform better than the present theoretical model when applied to predict the CFD data. The three simplified theoretical models based on Eqs. (15), (19) and (21) yield values of the uniform film thickness which differ from the CFD results by, respectively, $21 \%, 18 \%$ and $5.3 \%$, thus emphasizing the importance of utilizing the complete expression of the curvature in Eq. (20) in order to capture well the liquid film dynamics. 
It is worth noting that the theoretical model, the CFD results and the empirical correlations all show that, for a fixed $\mathrm{Ca}$, the uniform film thickness exhibits a decreasing trend with Re for Re $\lesssim 10^{2}$, followed by a monotonically increasing trend at larger Re numbers (Fig. 5). This behavior is observed also for the simpler theoretical models, e.g. Eq. (15) which includes only the viscous and inertial terms, and it originates from the expression of the inertial term. For Re $\ll 1$, the inertial term gives a negligible contribution to $Y_{X X X}$. As Re is increased above 1 , the inertial term gives an increasing negative contribution to $Y_{X X X}$ for $Y<\sqrt{6}$ (see Eq. (15)), and an increasing positive contribution to $Y_{X X X}$ for $Y>\sqrt{6}$. The overall behavior of $Y_{X X X}$ from $Y=1$ to $Y=H$ at each value of Re changes in such a way that, as $\operatorname{Re}$ is increased in the $\mathrm{Re} \lesssim 10^{2}$ range, lower values of $H$ are necessary in order to match the profile of the front meniscus to a sphere, while in the Re $\gtrsim 10^{2}$ range the values of $H$ must increase with Re. Since the inertial term is proportional to $\mathrm{Ca}^{1 / 3}$, the reduction of $H$ at intermediate values of the Reynolds number becomes more apparent at larger values of $\mathrm{Ca}$, as is suggested by the results shown in Fig. 5.

This mixed effect of inertia on the film thickness can be also derived by a scaling analysis of the forces acting on the front meniscus. Aussillous and Quéré [5] expressed the curvature of the bubble nose as

$$
\kappa \sim \frac{h_{0}}{\ell^{2}}+\frac{1}{R-h_{0}}
$$

where the $-h_{0}$ in the denominator of the second term accounts for the presence of the liquid film which increases the curvature of the bubble nose. However, inertia further increases the bubble nose curvature as it makes the bubble nose more slender. This effect can be accounted for by modifying Eq. (32) as

$$
\kappa \sim \frac{h_{0}}{\ell^{2}}+\frac{1+f_{\kappa}}{R-h_{0}}
$$

where $f_{\kappa}>0$ increases with both $\mathrm{Ca}$ and $\mathrm{Re}[3,8,9]$. Balancing the viscous force with the inertial force and the pressure gradient in the equation of motion along the dynamic meniscus yields

$$
\frac{\mu U}{h_{0}^{2}} \sim \frac{1}{\ell} \frac{\sigma\left(1+f_{\kappa}\right)}{R-h_{0}}-\frac{1}{\ell} \rho U^{2},
$$

while the matching between both static and dynamic menisci suggests

$$
-\frac{\sigma\left(1+f_{\kappa}\right)}{R-h_{0}}-\frac{\sigma h_{0}}{\ell^{2}} \sim-\frac{2 \sigma\left(1+f_{\kappa}\right)}{R-h_{0}} .
$$


Using Eq. (35) to replace $\ell$ in Eq. (34), a scaling law for the liquid film thickness is obtained:

$$
\frac{h_{0}}{R}=H \sim \frac{\mathrm{Ca}^{2 / 3}}{\mathrm{Ca}^{2 / 3}+\left(1+f_{\kappa}\right)^{1 / 3}\left(1+f_{\kappa}-\mathrm{We}^{\prime}\right)^{2 / 3}},
$$

where $\mathrm{We}^{\prime}=\rho U^{2}\left(R-h_{0}\right) / \sigma$. The positive function $f_{\kappa}$ in Eq. (36) acts to increase the denominator at low and intermediate Reynolds number values, while for larger values of Re the negative Weber number generates a monotonic increase of $H$ with Re.

\section{B. Numerical integration at the rear meniscus}

Numerical integration of Eq. (24) for the rear meniscus starts from close to point D and develops towards $X \rightarrow-\infty$. The linearized initial conditions now are written:

$$
\begin{aligned}
& Y(X=0)=1+\gamma \cos \varphi \\
& Y_{X}(X=0)=-\gamma\left[\alpha \cos \varphi+\frac{2 \pi}{\lambda} \sin \varphi\right], \\
& Y_{X X}(X=0)=\gamma\left[\alpha^{2} \cos \varphi+\frac{4 \pi}{\lambda} \sin \varphi-\frac{4 \pi^{2}}{\lambda^{2}} \cos \varphi\right],
\end{aligned}
$$

where we choose $\gamma=10^{-6}$. The nondimensional uniform film thickness $H$ is set to the value extracted from the numerical integration at the front meniscus. The solution now depends on the parameter $\varphi$, which shifts horizontally the profile characterizing the initial condition. Similarly to what was done for the front meniscus, here $\varphi$ is set to the value that allows matching the profile of the bubble back to a sphere (point E in Fig. 1(a)) at the farthest distance from the tube's wall. It will be shown in the next sections that this criterion yields theoretical profiles of the rear meniscus that compare quite well with the CFD results.

\section{RESULTS AND DISCUSSION}

CFD simulations and numerical integration of Eq. (24) (for both the front and rear menisci) are performed for a range of capillary numbers of $0.002 \leq \mathrm{Ca} \leq 0.1$ and Reynolds numbers of $10^{-1} \leq \operatorname{Re} \leq 10^{3}$. The CFD simulations are run with a bubble length of about $20 R$ that, as will be shown later on, is sufficient in order to treat the front and the rear menisci separately. In the next sections, a comparison of the rear menisci shapes obtained with the two methodologies is presented. The outcome of a systematic analysis of the 
parameters characterizing the ripples is then provided. Finally, the bubble length necessary to achieve the developed menisci profiles is discussed.

\section{A. Comparison of the rear meniscus profiles}

A comparison of the profiles of the bubble back obtained with the CFD simulations and the theoretical model is provided in Fig. 6 for some selected flow conditions. The test cases in (a), (b) and (c) are ordered by increasing the Weber number, We $=\mathrm{Ca}$ Re, which, as will be shown in Section VB, provides a better scaling of the inertial effects than the Reynolds number. A low Weber number case, $\mathrm{We}=0.005$, with $\mathrm{Re}=1$, is shown in Fig. 6(a). The agreement between the profiles is excellent and the differences are small. The undulation at the bubble tail exhibits one main crest (at $\left.X=X_{1}\right)$ and one main valley $\left(X-X_{1}=2.2-2.4\right)$. The CFD and the theoretical models yield similar values for the wavelength and amplitude of the ripples, and for the minimum film thickness value. Surprisingly, the theoretical model also captures very well the profile of the rear meniscus outside the film region, where the lubrication approximation is no longer valid.

Next, a low capillary $(\mathrm{Ca}=0.002)$ and high Reynolds number $(\mathrm{Re}=1000)$ case is shown in Fig. 6(b). The presence of two peaks and two valleys is now apparent. The two methodologies give very similar features of the undulations.

A relatively larger capillary $(\mathrm{Ca}=0.05)$ and Weber $(\mathrm{We}=5)$ number case is presented in Fig. 6(c). The case discussed has $\mathrm{Ca}^{2 / 3} \approx 0.14$ (model assumptions are valid as long as $\left.\mathrm{Ca}^{2 / 3} \ll 1\right)$, and the value of the Weber number suggests significant inertial effects. Here the difference between the undulation amplitude exhibited by the CFD simulations and the theoretical model is more evident, despite the good agreement observed for the wavelength. The results suggest that the model based on the numerical integration of Eq. (24) overestimates the effect of inertia, thus showing a larger amplitude of the ripple as the rear meniscus is approached. It is worth noting that the theory presently used includes inertia as a first order perturbation, and therefore is not necessarily suitable to describe flow configurations with significant inertial effects.

Finally, a systematic study of the effect of the Reynolds number on the profile of the bubble back is performed with the theoretical model, for a selected value of the capillary 


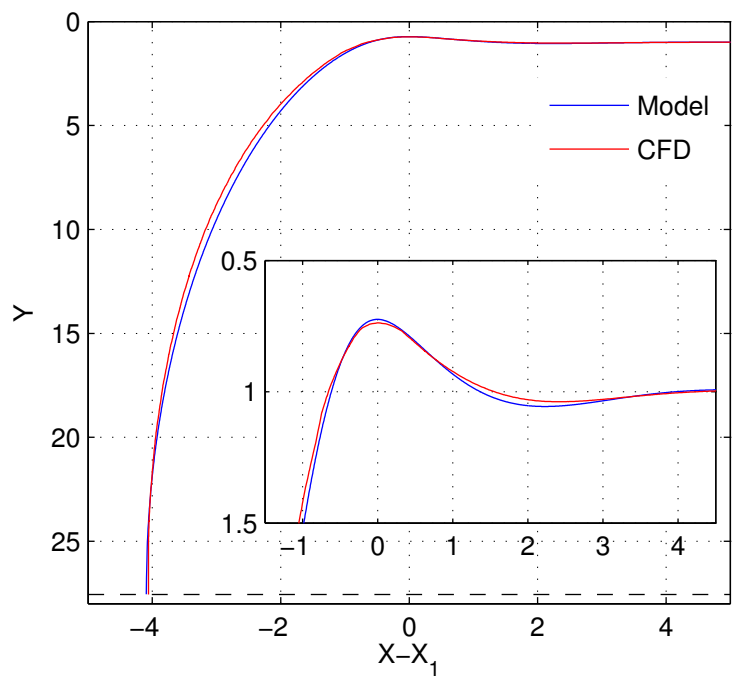

(a)

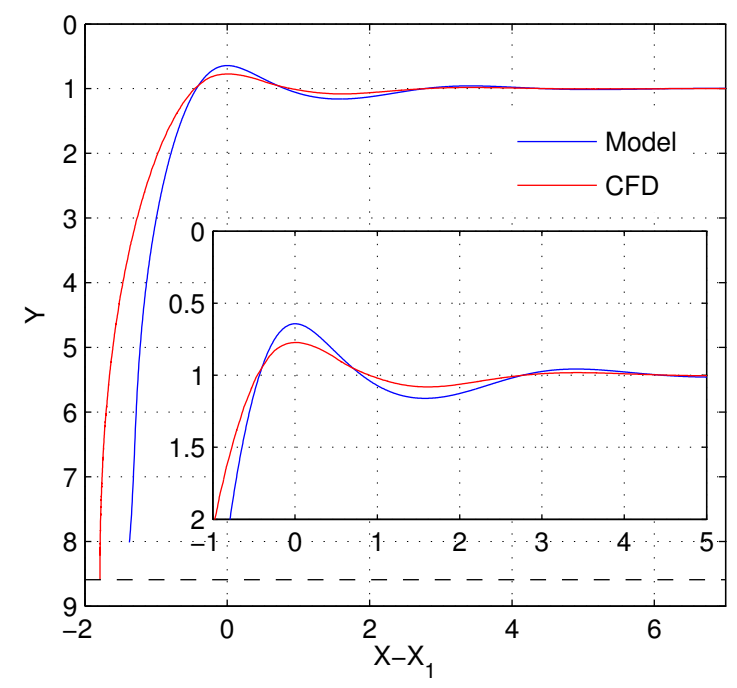

(c)

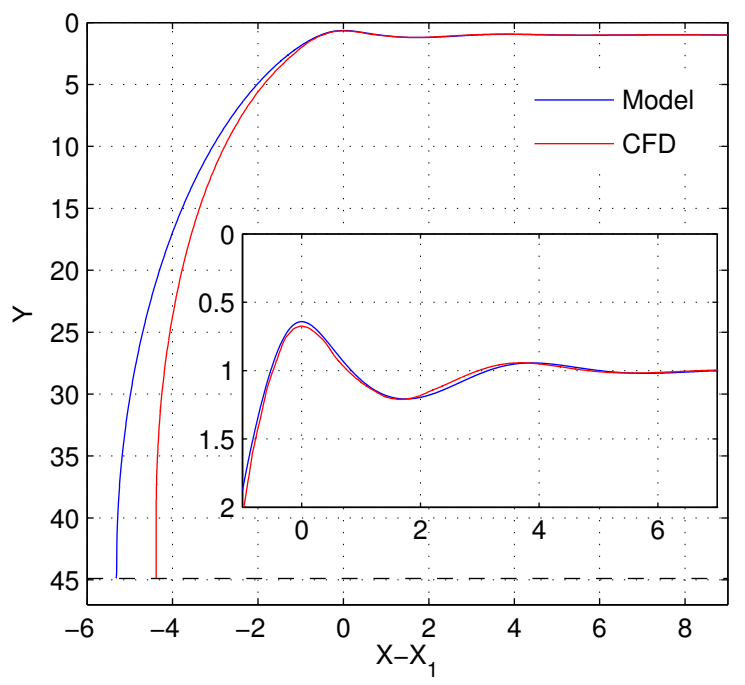

(b)

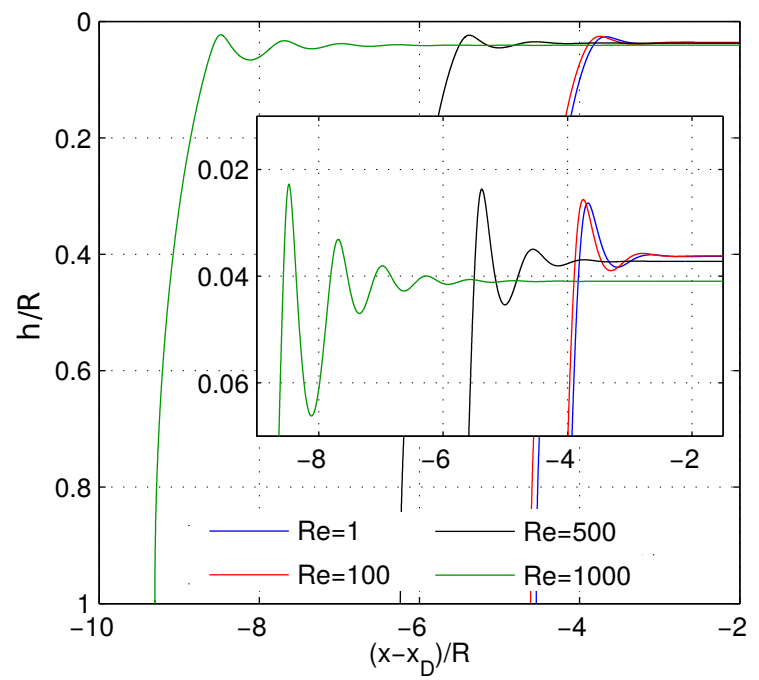

(d)

Figure 6. Comparison of the rear meniscus profiles obtained with the CFD simulations and by numerical integration of Eq. (24) for (a) $\mathrm{Ca}=0.005$ and $\mathrm{Re}=1$ (We $=0.005$ ), (b) $\mathrm{Ca}=0.002$ and $\mathrm{Re}=1000(\mathrm{We}=2)$, and $(\mathrm{c}) \mathrm{Ca}=0.05$ and $\mathrm{Re}=100(\mathrm{We}=5)$. The horizontal black dashed lines identify the location of the axis of the tube. $X_{1}$ locates the point of minimum film thickness at the rear meniscus (see Fig. 1(b)). (d) Profiles of the rear meniscus obtained by numerical integration of Eq. (24) for $\mathrm{Ca}=0.005$ and different values of the Reynolds numbers. $x_{D}$ locates the beginning of the dynamic meniscus region at the bubble back (see Fig. 1(a)). 
number, $\mathrm{Ca}=0.005$, as illustrated in Fig. 6(d). The main characteristics of the ripples when increasing Re are all well captured by the model. Similarly to Fig. 3, the liquid film profiles begin to change when $\operatorname{Re}>100$, i.e. We $\gtrsim 0.5$. The liquid film becomes thicker, and more undulation crests become apparent, while the minimum film thickness stays almost constant.

\section{B. Systematic analysis of the undulations}

The profiles of the rear meniscus displayed in Fig. 6 indicate that, when moving towards $X \rightarrow+\infty$, the undulation maintains a sinusoidal shape while its amplitude is gradually reduced. As such, its profile can be well approximated as a cosine function multiplied by an exponential damping term, analogous to the linear solution of Eq. (24) at the bubble back,

$$
Y(X)=1+\gamma e^{-\alpha X} \cos \left(\frac{2 \pi X}{\lambda}+\varphi\right),
$$

where $Y=h / h_{0}$ and $X=x /\left(h_{0} \mathrm{Ca}^{-1 / 3}\right)$. Here, $\lambda$ identifies the wavelength of the undulation, while $\alpha$ represents its decay rate, both in nondimensional units; $\alpha>0$ in order for the amplitude to decrease in the direction of increasing $X$. Estimates of $\lambda$ and $\alpha$ as a function of the capillary and Reynolds numbers are already provided as roots of Eq. (26).

The wavelength and decay rate in actual rear meniscus shapes predicted by the CFD simulations and by the numerical integration of Eq. (24) are extracted as illustrated in Fig. 1(b). The ripple on the bubble surface is assumed to be well represented by Eq. (38). $\left(X_{1}, Y_{1}\right)$ denote the nondimensional coordinates of the most upstream crest, which is also the largest amplitude. Also, $\left(X_{2}, Y_{2}\right)$ indicate the coordinates of the second crest along the positive $X$ direction. The wavelength is calculated as the nondimensional distance between these two most upstream crests

$$
\lambda=X_{2}-X_{1}
$$

Note that in the dimensional units based on the lubrication description, this distance is $x_{2}-x_{1}=\lambda h_{0} \mathrm{Ca}^{-1 / 3}$, where $\lambda$ is the nondimensional wavelength given by Eq. (39). According to Eq. (38), the decay rate of the undulation is calculated by considering the decrease of its nondimensional amplitude between the first and second most upstream crests

$$
\alpha=\frac{1}{\lambda} \ln \left(\frac{1-Y_{1}}{1-Y_{2}}\right),
$$


and its value in dimensional units is calculated as $\alpha /\left(h_{0} \mathrm{Ca}^{-1 / 3}\right)$, where $\alpha$ is obtained from Eq. (40).

The results obtained from the systematic analysis of the undulations are presented below as a function of the capillary and Weber numbers. For this problem with interfacial dynamics we have observed that the Weber number is more effective than the Reynolds number in unifying trends and magnitudes when inertial effects become relevant. Moreover, the Weber number can be interpreted as an effective Reynolds number in thin film flows, as it represents the effective ratio between inertia, acting along the streamwise direction, and viscous effects, which act along a direction orthogonal to that of the flow, i.e. from the momentum equation we note

$$
\frac{\frac{\partial(\rho u u)}{\partial x}}{\mu \frac{\partial^{2} u}{\partial y^{2}}} \approx \frac{\frac{\rho U^{2}}{\ell}}{\frac{\mu U}{h_{0}^{2}}}=\frac{\rho U}{\mu} \frac{h_{0}^{2}}{\ell}=\frac{\rho U}{\mu} h_{0} \mathrm{Ca}^{1 / 3} \approx \frac{\rho U}{\mu} R \mathrm{Ca}^{2 / 3} \mathrm{Ca}^{1 / 3}=\mathrm{Re} \mathrm{Ca}=\mathrm{We}
$$

where basic results of the Bretherton problem have been utilized; the capillary effects enter in the expression above because they determine the thickness of the liquid layer, i.e. the characteristic length along which viscous effects act. It is common to interpret We as the ratio of inertial stresses to capillary stresses. Nevertheless, as Eq. (41) suggests, for thin film flow where the local pressure gradient is set by capillary effects, as in the Bretherton problem, then the ratio of inertial effects to viscous effects is also set by the Weber number.

Now we report the wavelength of the ripples as a function of the Weber number in Fig. 7(a), for different values of Ca. Results from the CFD simulations, from numerical integration of Eq. (24) and the values obtained from the solution of the characteristic polynomial Eq. (26) are included in the same graph. It can be seen that the data cluster nicely when reported against We. Trends are approximately independent of $\mathrm{Ca}$ as long as $\mathrm{Ca} \leq 0.05$. The CFD and the theoretical model (both the linear and complete solutions) agree very well up to $\mathrm{We} \approx 10$, when time-dependent patterns are detected in the numerical simulations (shaded area in Fig. 7(a)). It is interesting to note that the wavelength obtained from the linear model is always very close to the values extracted from the complete theoretical model, despite being slightly higher when $\mathrm{We}<1$.

As general trends, when We $<10^{-1}$ the results collapse onto a single curve for Ca up 
to 0.05 and are independent of the Weber number. This suggests that inertial effects are negligible for this parameter range. Within this range, the nondimensional wavelength has the value $\lambda=4.7-4.8$. In the asymptotic limit of $\mathrm{Ca}^{1 / 3} \ll 1$, Bretherton [1] obtained the value of $\lambda=5.03$ from the linear solution of Eq. (5), and a value of $\lambda=4.82$ is extracted from the profile obtained from the numerical integration of Eq. (5).

When We $>10^{-1}$ the inertial effects manifest with a decrease of the wavelength as the Weber number increases, which is in line with the results obtained by Giavedoni and Saita [14] and Edvinsson and Irandoust [3] by means of numerical simulations, while the capillary number has a negligible effect. de Ryck and Quéré [28] observed that in fibre coating the thickness of the liquid film began to deviate from the low Reynolds number theory when We $\approx 1$, which is the value typically assumed in the practice to judge the importance of inertial effects. The present results suggest that inertial effects begin to appear when $\mathrm{We}>10^{-1}$, and become evident when $\mathrm{We} \simeq 1$.

When We $>10$, time-dependent patterns appear in the CFD simulations analogously

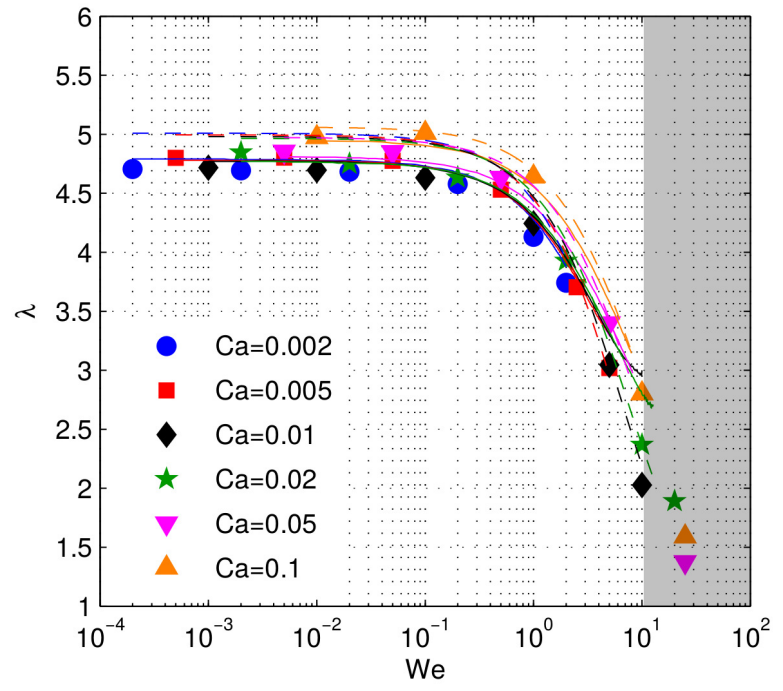

(a)

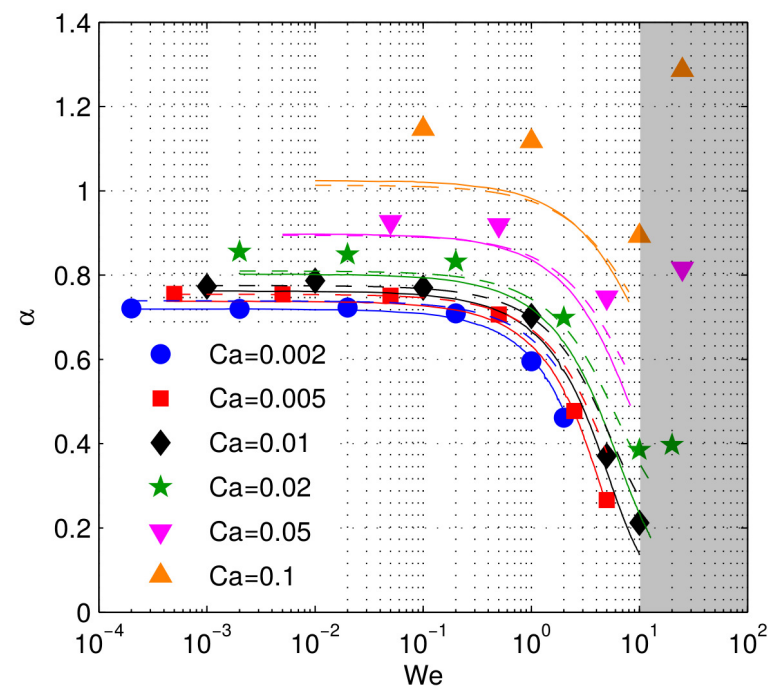

(b)

Figure 7. (a) Wavelength and (b) decay rate of the ripples appearing at the rear meniscus of a bubble extracted from the CFD results (symbols), from numerical integration of Eq. (24) (solid lines) and from the solution of the linear Eq. (26) (dashed lines). The shaded area indicates the presence of time-dependent patterns in the CFD simulations. 
to what was observed both experimentally and numerically by Khodaparast et al. [9]. The wavelength becomes a function of time and the values reported in Fig. 7(a) have to be interpreted as time-averaged quantities. We $\approx 10$ represent a limiting condition also for the theoretical model. Above this critical value (actually for values of We that slightly decrease when increasing $\mathrm{Ca}$ ), the numerical integration of Eq. (24) towards the bubble back yields oscillations that grow unbounded as $X \rightarrow-\infty$, thus making it impossible to match the profile to a sphere.

The decay rate of the ripples as a function of the Weber number is presented in Fig. 7(b), for different values of $\mathrm{Ca}$. It can be seen that the capillary number is now an influential parameter, as larger values increase the decay thus making the amplitude of the ripples decrease rapidly. Therefore, the oscillation tends to disappear after the first crest and valley, and the existence of a smooth and long liquid film region around the bubble is promoted. This trend is in agreement with results reported by Giavedoni and Saita [14]. The theoretical model presented here compares quite well with the CFD simulations up to $\mathrm{Ca}=0.05$, although it systematically underestimates the decay rate. Relatively larger deviations are seen for $\mathrm{Ca}=0.1$, although they are always below $15 \%$, which was already noticed when comparing the profiles of the bubble rear meniscus at large capillary and Weber numbers (Fig. 6(c)), and can be attributed to the limited validity of the model assumptions as the value of the capillary number approaches 0.1 .

As a general trend, as the Weber number is decreased below $10^{-1}$ all the curves tend to asymptotic values dependent on the capillary number. For $\mathrm{Ca}=0.002$, this asymptotic value is $\alpha=0.72$, which matches quite well the value obtained by Bretherton [1], $\alpha=0.696$ when considering the bubble profile integrated from Eq. (5), while $\alpha=0.72$ when considering the linear solution of Eq. (5).

Inertial effects make the trends dependent on the Weber number when We $>10^{-1}$. The spatial decay of the undulations decreases, and therefore more crests and valleys become visible, as was already observed in previous works $[9,29]$ and in agreement with the trends in Fig. 3.

When time-dependent patterns occur in the CFD simulations (We $\geq 10$ ), the timeaveraged values of $\alpha$ reported in Fig. 7(b) are not really meaningful because they are characterized by a very large standard deviation, which is on the order of the mean value itself. 
Furthermore, the amplitude of the second crest can become larger than that of the first crest at certain time instants, thus making $\alpha$ negative. The analysis of this time-dependent regime for the flow of the bubbles is outside of the scope of the present paper.

The average deviation between the CFD results and theoretical model based on Eq. (24) for all of the data points included in Fig. 7 is $4.4 \%$ and $10.9 \%$ for the wavelength and decay rate, respectively. The simplified models exhibit the following deviations, respectively, for the wavelength and decay rates: Eq. (15), $9.8 \%$ and $17 \%$; Eq. (19), $9.8 \%$ and $15.7 \%$; Eq. (21), $6.3 \%$ and $13.1 \%$. These results emphasize once more that the use of the complete expression for the curvature, Eq. (20), yields a significant improvement of the description of the liquid film profile.

\section{Minimum bubble length}

It has been observed both in Fig. 3 and Fig. 6(d) that the length of the dynamic meniscus at the bubble back increases with the Reynolds number. Figure 7(a) suggests that the dimensional wavelength $\left(\sim \mathrm{Ca}^{1 / 3}\right)$ increases with the capillary number, thus making the dynamic meniscus longer. An increase of the transition region with increasing $\mathrm{Ca}$ and $\mathrm{Re}$ was already reported in the studies of de Ryck [6] for the front meniscus of bubbles in the visco-inertial regime, and by Cherukumudi et al. [12] for the entire bubble (front plus rear menisci) in the $R e \ll 1$ regime.

When running CFD simulations or experiments with a finite bubble length, if the bubble is not long enough a flat liquid film region is not recovered, which means that the front and rear menisci cannot be considered separately. The consequence is that the measured thickness of the liquid film in such conditions depends on the bubble length. The measured value decreases with the bubble length, down to a constant value that is reached when the bubble is sufficiently long. This feature may affect the comparison of data from independent studies, if the actual bubble length adopted for each liquid film thickness measurement is not provided. This issue was already raised by Khodaparast et al. [9] who observed that in experiments with water and air at high Reynolds number their bubbles of length $6 R$ were not showing any flat film region, while associated CFD simulations showed that only bubbles much longer than $6 R$ satisfied this requirement.

Therefore, the knowledge of the minimum bubble length necessary for the complete de- 
velopment of front and rear menisci has practical importance. To this end, we used the theoretical model based on the numerical integration of Eq. (24) to estimate the length of the transition regions in the range of capillary and Reynolds number of interest for this study.

The inset in Fig. 8(a) shows a sketch of the procedure for the calculation of the length at the front meniscus. This length is evaluated as the axial distance between the tip of the bubble nose (point A) and the location where the liquid film thickness $h$ decreases below 1.01 times the uniform film thickness $h_{0}$. The inset in Fig. 8(b) illustrates the procedure for the bubble back. The length of the dynamic region of the rear meniscus is taken as the axial distance between the tip of the bubble back (point F) and the point where the liquid film thickness deviates from $h_{0}$ by less than $1 \%$. The $1 \%$ threshold for both nose and tail is a small value that was chosen arbitrarily.

The plots of the front meniscus and of the entire bubble (front plus rear) length are given in Fig. 8. The curves are presented versus the Reynolds number because this is still more frequently used than the Weber number when reporting results for confined bubble flows in narrow channels.

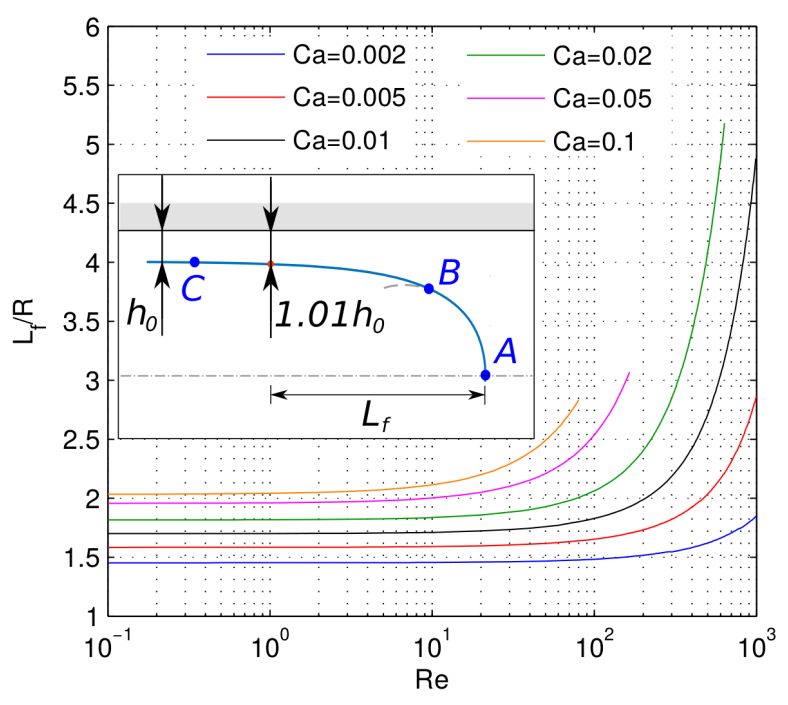

(a)

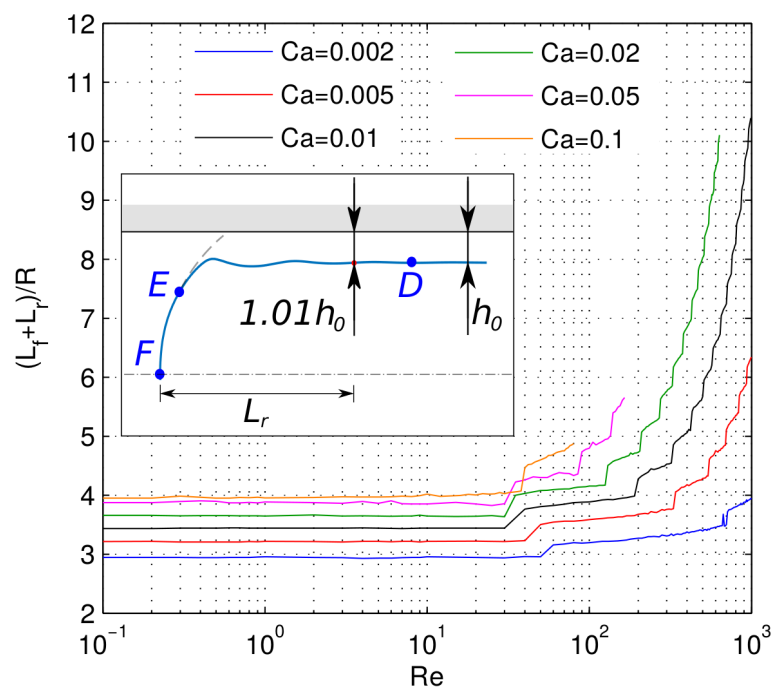

(b)

Figure 8. Minimum length of the (a) front meniscus and (b) entire bubble necessary to recover a flat film region, calculated by numerical integration of Eq. (24). The insets illustrate the criterion adopted to evaluate the length of the transition regions. 
When inertial effects are absent, the transition length is a function of the capillary number only and it increases with Ca. As $\mathrm{Ca} \rightarrow 0, L_{f} / R \rightarrow 1$ and $L_{b} / R \rightarrow 2\left(L_{b}=L_{f}+L_{r}\right)$, i.e. the bubble exhibits spherical caps. In these conditions, the transition region at the bubble back is slightly longer than that at the front due to the presence of an undulation at the matching point between the dynamic and static menisci. The minimum bubble length in this visco-capillary regime is always relatively short, below $4 R$ up to $\mathrm{Ca}=0.1$.

Inertial effects appear at decreasing values of the Reynolds number when increasing Ca (at We $\approx 10^{-1}$ ). As is evident in Fig. 8(b), the necessary length increases steeply with Re, in particular at high $\mathrm{Ca}$, and it grows above $10 R$ when the Reynolds number approaches 1000. Note that curves for $\mathrm{Ca} \geq 0.02$ are interrupted when the matching of the rear meniscus to a sphere is no longer possible, which also corresponds to the onset of time-dependent patterns in the CFD simulations. Air bubbles flowing in water within a tube of diameter of $0.5 \mathrm{~mm}$ at $\mathrm{Ca}=0.02$ have Re of about 900. Figure 8(b) demonstrates that bubbles with length $6 R$, such as those used in the work of Khodaparast et al. [9], are not sufficient to recover a flat film region under such conditions.

The steps in the curves in Fig. 8(b) at high Re reveal the appearance of new crests on the surface of the bubble at the rear meniscus, i.e. oscillations whose amplitude has grown above $0.01 h_{0}$. It can be seen that a second crest appears at $\operatorname{Re}=35-60$, which corresponds to Weber numbers that range from 0.12 (at $\mathrm{Ca}=0.002)$ to $4(\mathrm{Ca}=0.1)$. The appearance of the third crest is anticipated as the capillary number is increased, which happens for We ranging from $1.42(\mathrm{Ca}=0.002)$ to $4.5(\mathrm{Ca}=0.05)$.

\section{CONCLUSIONS}

In this study we present a systematic analysis of the undulations appearing on the surface of long bubbles in confined gas-liquid flows. CFD simulations are utilized to generate bubble profiles in the capillary number range of $0.002-0.1$ and Reynolds number range of $0.1-1000$. A theoretical model based on the extension of the classical axisymmetric Bretherton theory accounting for inertia and for the curvature of the tube's wall is adopted to help understand the CFD results. The profiles of the rear meniscus of the bubble obtained by the CFD simulations and by the theoretical model agree reasonably well across all the range of $\mathrm{Ca}$ and Re numbers of interest. The results indicate that the evolution of the undulations is 
well described by the fluid mechanics of the thin liquid film surrounding the bubble, in the presence of inertial effects. When the Weber number of the flow $\mathrm{We}=\mathrm{Ca} \operatorname{Re} \lesssim 10^{-1}$, inertial effects are negligible and the wavelength of the only visible undulation asymptotically

converges to the value derived from the low Reynolds number flow theory. When We $\geq 1$, inertial effects are significant with a monotonic decrease of the wavelength of the ripples. The undulations decay less towards the direction of the bubble front and more crests become evident, as observed in previous studies. Time-dependent patterns appear in the CFD simulations when We $>10$, and therefore the present study is limited to values of the Weber number of 10 and below. The lengths of the dynamic menisci at the front and rear of the bubble increase mildly with $\mathrm{Ca}$ and steeply with $\mathrm{Re}$ (when Re $>100$ ). As a result, the length that the bubble should have in order for a flat liquid film region to exist can quickly grow above five tube diameters when the Reynolds number approaches 1000.

\section{Appendix A: Equations governing the flow in the liquid film}

The equations governing the flow in the liquid film region surrounding the bubble, in the cylindrical axisymmetric coordinates introduced in Fig. 1(a), are written:

$$
\begin{aligned}
& u_{x}+v_{y}-\frac{v}{R-y}=0 \\
& \rho\left(u u_{x}+v u_{y}\right)=-p_{x}+\mu\left(u_{x x}+u_{y y}-\frac{u_{y}}{R-y}\right) \\
& \rho\left(u v_{x}+v v_{y}\right)=-p_{y}+\mu\left[v_{x x}+v_{y y}-\frac{v_{y}}{R-y}-\frac{v}{(R-y)^{2}}\right] .
\end{aligned}
$$

When neglecting the viscous stress within the gas phase, and setting the pressure within the bubble to a zero reference value, the boundary conditions at the free interface are

$$
\begin{aligned}
& u_{y}+v_{x}+\frac{2 h_{x}}{1-h_{x}^{2}}\left(v_{y}-u_{x}\right)=0, \quad \text { at } y=h(x), \\
& \text { and } p+\sigma \kappa+\mu h_{x}\left(u_{y}+v_{x}\right)-2 \mu v_{y}=0, \quad \text { at } y=h(x) .
\end{aligned}
$$

The normal vector at the interface has components

$$
\boldsymbol{n}=\frac{1}{\left(1+h_{x}^{2}\right)^{1 / 2}}\left(-h_{x}, 1\right)
$$

and the interface curvature is expressed as shown in Eq. (20). 


\section{Appendix B: Supplemental Material}

Supplemental Material associated with this article, including details of the derivation of the theoretical models briefly introduced in Section IV, is available in the online version.

\section{ACKNOWLEDGMENTS}

M. M. is supported by the Swiss National Science Foundation (SNSF) under Contract No. 200020-156181. The authors are grateful to G. Balestra, G. Gallino and Prof. F. Gallaire (Laboratory of Fluid Mechanics and Instabilities, EPFL) for valuable discussions and comments during the development of this work.

[1] F. P. Bretherton, "The motion of long bubbles in tubes," J. Fluid Mechanics 10, 166-188 (1961).

[2] G. I. Taylor, "Deposition of a viscous fluid on the wall of a tube," J. Fluid Mechanics 10, 161-165 (1961).

[3] R.K. Edvinsson and S. Irandoust, "Finite-element analysis of Taylor flow," AIChE J. 42, 1815-1823 (1996).

[4] M. D. Giavedoni and F. A. Saita, "The axisymmetric and plane cases of a gas phase steadily displacing a Newtonian liquid - A simultaneous solution of the governing equations," Physics of Fluids 9, 2420-2428 (1997).

[5] P. Aussillous and D. Quéré, "Quick deposition of a fluid on the wall of a tube," Physics of Fluids 12, 2367-2371 (2000).

[6] A. de Ryck, "The effect of weak inertia on the emptying of a tube," Physics of Fluids 14, $2102-2108$ (2002).

[7] Y. Han and N. Shikazono, "Measurement of the liquid film thickness in microtube slug flow," Int. J. Heat and Fluid Flow 30, 842-853 (2009).

[8] M. T. Kreutzer, F. Kapteijn, J. A. Moulijn, C. R. Kleijn, and J. J. Heiszwolf, "Inertial and interfacial effects on pressure drop of Taylor flow in capillaries," AIChE Journal 51, 2428-2440 (2005). 
[9] S. Khodaparast, M. Magnini, N. Borhani, and J. R. Thome, "Dynamics of isolated confined air bubbles in liquid flows through circular microchannels: an experimental and numerical study," Microfluid. Nanofluid. 19, 209-234 (2015).

[10] M. Heil, "Finite Reynolds number effects in the Bretherton problem," Physics of Fluids 13, $2517-2521$ (2001).

[11] D. R. Langewisch and J. Buongiorno, "Prediction of film thickness, bubble velocity, and pressure drop for capillary slug flow using a CFD-generated database," Int. J. Heat and Fluid Flow 54, 250-257 (2015).

[12] A. Cherukumudi, E. Klaseboer, S. A. Khan, and R. Manica, "Prediction of the shape and pressure drop of Taylor bubbles in circular tubes," Microfluid. Nanofluid. 19, 1221-1233 (2015).

[13] J. Ratulowski and H.-C Chang, "Transport of gas bubbles in capillaries," Physics of Fluids A 1, 1642-1655 (1989).

[14] M.D. Giavedoni and F.A. Saita, "The rear meniscus of a long bubble steadily displacing a Newtonian liquid in a capillary tube," Physics of Fluids 11, 786-794 (1999).

[15] S. Khodaparast, M. K. Kim, J. Silpe, and H. A. Stone, "Bubble-driven detachment of bacteria from confined micro-geometries," Environmental Science \& Technology 51, 1340-1347 (2017).

[16] M. Magnini and J. R. Thome, "A CFD study of the parameters influencing heat transfer in microchannel slug flow boiling," Int. J. Thermal Sciences 110, 119-136 (2016).

[17] N. Borhani, B. Agostini, and J. R. Thome, "A novel time strip flow visualization technique for investigation of intermittent dewetting and dryout in elongated bubble flow in a microchannel evaporator," Int. J. Heat and Mass Transfer 53, 4809-4818 (2010).

[18] N. Borhani and J. R. Thome, "Intermittent dewetting and dryout of annular flows," Int. J. Multiphase Flow 67, 144-152 (2014).

[19] C. W. Hirt and B. D. Nichols, "Volume of fluid (VOF) method for the dynamics of free boundaries," J. Computational Physics 39, 201-225 (1981).

[20] J. U. Brackbill, D. B. Kothe, and C. Zemach, "A continuum method for modeling surface tension," J. Computational Physics 100, 335-354 (1992).

[21] B. Lafaurie, C. Nardone, R. Scardovelli, S. Zaleski, and G. Zanetti, "Modelling merging and fragmentation in multiphase flows with SURFER," J. Computational Physics 113, 134-147 (1994). 
[22] A. Ferrari, M. Magnini, and J. R. Thome, "A Flexible Coupled Level Set and Volume of Fluid (flexCLV) method to simulate microscale two-phase flow in non-uniform and unstructured meshes," Int. J. Multiphase Flow 91, 276-295 (2017).

[23] S. V. Patankar, Numerical Heat Transfer and Fluid Flow (Hemisphere Publishing, New York, 1980).

[24] V. Ya. Shkadov, "Wave flow regimes of a thin layer of viscous fluid subject to gravity," Izv. Akad. Nauk URSS Mekh. Zh. Gaza 2, 43-51 (1967).

[25] M. N. Esmail and R. L. Hummel, "Nonlinear theory of free coating onto a vertical surface," AIChE J. 21, 958-965 (1975).

[26] A. Koulago, V. Shkadov, D. Quéré, and A. de Ryck, "Film entrained by a fiber quickly drawn out of a liquid bath," Physics of Fluids 7, 1221-1224 (1995).

[27] A. de Ryck, "Erratum: The effect of weak inertia on the emptying of a tube," Physics of Fluids 14, 4100 (2002).

[28] A. de Ryck and D. Quéré, "Inertial coating of a fibre," J. Fluid Mechanics 311, 219-237 (1996).

[29] M. Magnini, B. Pulvirenti, and J. R. Thome, "Numerical investigation of hydrodynamics and heat transfer of elongated bubbles during flow boiling in a microchannel," Int. J. Heat and Mass Transfer 59, 451-471 (2013). 